\title{
Depression and Anxiety Analysis from Elder Population in A Beijing Rural Area
}

\author{
Yin Zhang1, Chuang Shi ${ }^{2}$ and Yinyun $\mathrm{Li}^{3 *}$ \\ ${ }^{1}$ Institute of Medical Information, Chinese Academy of Medical Science, Beijing, 100020, China \\ ${ }^{2}$ School of Government Management, Beijing Normal University, Beijing, 100875, China \\ ${ }^{3}$ School of Systems Science, Beijing Normal University, Beijing, 100875, China \\ *Correspondence author: Yinyun Li, School of Systems Science, Beijing Normal University, Beijing, 100875, China; Email: Yinyun@bnu.edu.cn
}

Received: June 10, 2019; Accepted: June 20, 2019; Published: June 22, 2019;

\begin{abstract}
The paper aims to investigate the mental health status of elderly people in Beijing rural area, and look for possible factors that affect the depression and anxiety levels. 204 ordinary elderly residents over 60 years old without major diseases are randomly selected from a rural area of Beijing. The factors influencing the level of depression and anxiety of the sample are investigated by the Daily Living Ability Scale, the Marital Attachment Questionnaire and the Social Support Rating Scale. Our results show that the degree of depression is influenced by 12 factors (including age, marriage, education, monthly income, source of income, type of health care, daily living ability, number of chronic diseases, couple attachment, parent-child contact, social support and frequency of Internet use), among which the top three most significant factors are social support, marriage and couple attachment. The degree of anxiety is generally influenced by 10 factors (age, marriage, education, monthly income, source of income, daily living ability, number of chronic diseases, couple attachment, social support and frequency of Internet use), among which the top three most significant factors are the number of chronic diseases, age and daily living ability. Our results indicate that the depression level is affected by the relationship between oneself and other people; while the anxiety degree is mainly affected by the basic daily life and relationship between oneself. Our research results suggest that, the society shall focus on especially the vulnerable elderly groups with poor daily living ability and many chronic diseases, and efforts should be made to optimize primary medical care services for the aged population; family and society emotional support for the elderly should be strengthened to relieve the depression level.
\end{abstract}

Keywords: Mental Health; Depression; Anxiety; Elderly People; Influencing Factors; Uni-variable Analysis; Dependent Variable; Independent Variable; SPSS

\section{Preface}

Aging is a principle trend of population development in the world today. In China, one of the most populous countries in the world, the problem of population aging began to appear gradually since the 1980s, and showed accelerating velocity nowadays. The aggravation of population aging has an important impact on the function and management of the society. In comparison to the developed countries in the western world, the aged population in China has a large base, which develops even rapidly, while the system for elderly care and medical service is obviously lagging behind. With the development of social environment, the family structure also has a rapid transformation, weakening the original intergenerational support and care ability. This requires the elderly not only to deal with the physiological and psychological pressure brought by their aging but also to face the problems including insufficiency of family and social support, which lead them easily to fall into depression, anxiety and other psychological problems [1-4]. The mental health problem of the elderly not only bring painful experience to themselves and their families, but also increase the economic burden of the whole society and eventually slow down the development, therefore it becomes to be a more and more severe social problems and cannot be ignored.
This paper focuses on two basic mental health problems: depression and anxiety, and attempts to find out the main factors that affect them.

The research of mental health of the elderly began much earlier in western countries, which divided the factors affecting the mental health of the elderly mainly into demographic factors, socio-economic factors, lifestyle factors, diseases and medical and health care factors [5-9].

From the domestic research in China, Wu Zhenyun pointed out that the mental health of the elderly is affected by factors which can generally be summarized as objective factors and subjective factors [10]. Objective factors mainly include the demographic characteristics and health status of the elderly number of diseases, number of life events and other social factors (such as family, economic status, etc.) Subjective factors include mainly a variety of measures on feeling of satisfaction and happiness [10,11]. Besides, Yang Jinging addressed that intergenerational relationship has an important impact on the mental health of the elderly, and a harmonious and intimate intergenerational relationship helps to improve the degree of happiness and satisfaction for the elder population $[5,12]$.

The sample of the research object is generally selected in a certain region, and questionnaire survey is carried out for the data collection, 
then the empirical and quantitative analysis is implemented [10, 13-15]. In this work, the same working paradigm is applied, and the research content mainly includes the following sections. Firstly, we evaluate of the present situation of depression and anxiety levels of the elderly by the survey result and questionnaire analysis; secondly, we analyze the influencing factors of the depression and anxiety of the elderly and find out the sequence of significance of affecting factors and differentiate the affecting factors for depression and anxiety; finally we suggest potential policies and possible strategy to improve the mental health level of the elderly.

\section{Objects and Methods}

As the conceptions of mental health are difficult to quantify, international experts have developed certain scales to measure mental health, such as the Symptom CheckList-90(SCL-90) [6], Self-rating Depression Scale [7,8], Activities of Daily Living Scale (ADL) [9]. Research on mental health in China started in the 1960s and the scales developed by domestic experts include Social Support Rating Scale (SSRS) [16],Daily Living Ability Scale [3], mental health questionnaire for the elderly [10] and couple relationship questionnaire [5]. These scales and questionnaires are abstracted and simplified by the researchers and easy to carry out, which greatly promote the research process. In this work, both international and domestic scales and questionnaires have been adopted and integrated.

To better understand the mental health status of elderly, 204 ordinary elderly local residents without major diseases (from 60 to 84 years old) are randomly selected from a rural area of Beijing with a well-off level of economic status as research objects. The household questionnaire survey has been carried out from door to door and interviews were made. The content of the questionnaire mainly contains questions related to mental health measurement (GDS and SAS) and factors affecting depression and anxiety levels, including demographic factors, economic basis, physiological health condition, living environment support and new factors. In specific, demographic factors include gender, age, marriage and education. Economic basis is measured by monthly income, source of income and type of medical insurance. Physical health condition is measured by daily living ability and the number of chronic diseases. Living environment support includes couple attachment, parent-child contact and social environment support. The frequency of Internet use is considered as the new factor. The scales used mainly include Daily Living Ability Scale, Couple Attachment Questionnaire and Social Support Rating Scale (SSRS). The results from questionnaire are collected into Excel and processed by SPSS software.

\section{Results}

\section{Basic Results}

The statistical data of basic conditions of the sample and the scores of depression and anxiety are shown in (Table 1). The proportion of male in the survey is slightly higher than that of female. The results show that the average score of depression in the elderly is $10.42 \pm$ 9.712 , and the average score of anxiety is $49.23 \pm 16.468$. Among them, $60.3 \%$ of the population is normal without depression, $22.5 \%$ is mild depressed, and $17.2 \%$ is moderate-severe depressed. On the other hand, $56.4 \%$ of the population is normal without anxiety, $19.6 \%$ is with mild anxiety, $8.8 \%$ is with moderate anxiety, and $15.2 \%$ is with severe anxiety, as can be seen in Table 1 .

\section{The Influence of Demographic Characteristics on Depression and Anxiety of the Elderly}

It can be seen from table 1 that age, marital status and education have significant influence on the degrees of depression and anxiety. With the growth of age, the depression degree of the elderly tends to deepen, while the anxiety degree declines in the middle but generally becomes more severe. Specifically, the depression scores of the elderly in the two stages of 65-69 and 70-74 years old are almost the same. However, age 75 seems to be a dividing line, people older than 75 years old have their scores of depression and anxiety significantly higher than those who are younger than 75 . In terms of marital status, people who have a spouse but his/her spouse is already dead have the highest level of anxiety, scoring even higher than those who never have a spouse. To certain extent, our result indicates that the death of a spouse increases the feeling of loneliness and anxiety. The highest score of depression is found in the elderly without a spouse, whose average score is significantly higher than those with a spouse (regardless of whether the spouse is alive or not), indicating that the level of depression is significantly increased in the elderly living without a spouse. From the aspect of education, with the improvement of the education level, the level of depression and anxiety tends to decline. Among them, the elderly who have never attended school account for about $15 \%$, and their anxiety and depression degrees are significantly higher than those who have received primary education and above. Primary education can be seen as a dividing line. The scores of anxiety and depression of the elderly group with primary and junior middle school education are similar without significant difference. The depression score of the elderly with senior high school education is significantly lower than those with primary and middle school education.

\section{The Influence of Economic Basis on Depression and Anxiety Status of the Elderly}

Monthly income, source of income and type of medical insurance have significant influence on depression degree of the elderly, while monthly income and source of income have significant influence on anxiety degree of the elderly. With the increase of monthly income, the depression degree of the elderly tends to decrease, while the anxiety degree increases in the middle but generally declines. It is worth pointing out that the degrees of anxiety and depression in the elderly group with a monthly income of less than 1,000 yuan are significantly enhanced. It may indicate that monthly income of 1,000 yuan can provide basic living security, while there is basically no difference in anxiety scores of the elderly group with a monthly income of 1000 3000 and 3000-5000. With different sources of income, the elderly have different degrees of depression: when the economic source is not from oneself, the depression and anxiety levels of the elderly tend to increase. It may suggest that the income dependency on children or the society would induce certain strange and complex psychological feelings, a certain amount of guilt, insecurity and so on, which could 
Yin Zhang, Chuang Shi, Yinyun Li (2019) Depression and Anxiety Analysis from Elder Population in A Beijing Rural Area

Table 1. Statistical results of basic conditions and scores of depression and anxiety of the elderly.

\begin{tabular}{|c|c|c|c|c|c|}
\hline \multicolumn{2}{|r|}{ Item } & \multirow{2}{*}{ Amount } & \multirow{2}{*}{$\begin{array}{c}\text { Percentage } \\
30.9 \%\end{array}$} & \multirow{2}{*}{$\begin{array}{c}\text { Average score of depression } \\
6.70 \pm 8.462\end{array}$} & \multirow{2}{*}{$\begin{array}{c}\text { Average score of anxiety } \\
40.38 \pm 11.715\end{array}$} \\
\hline Age & $1(60 \sim 64)$ & & & & \\
\hline & $2(65 \sim 69)$ & 71 & $34.8 \%$ & $9.17 \pm 9.307$ & $47.15 \pm 12.478$ \\
\hline & $3(70 \sim 74)$ & 14 & $6.9 \%$ & $9.93 \pm 8.810$ & $42.36 \pm 12.659$ \\
\hline & $4(75 \sim 79)$ & 35 & $17.2 \%$ & $14.40 \pm 9.802$ & $60.11 \pm 14.766$ \\
\hline & $5(80+)$ & 21 & $10.3 \%$ & $19.52 \pm 7.366$ & $69.24 \pm 19.611$ \\
\hline \multirow[t]{2}{*}{ Gender } & 1 (male) & 106 & $52.0 \%$ & $11.12 \pm 10.476$ & $47.39 \pm 16.493$ \\
\hline & 2 (female) & 98 & $48.0 \%$ & $9.66 \pm 8.801$ & $51.22 \pm 16.289$ \\
\hline \multirow[t]{3}{*}{ Marriage } & 1 (married and the spouse is alive) & 141 & $69.1 \%$ & $7.00 \pm 7.727$ & $45.77 \pm 14.005$ \\
\hline & 2 (married but the spouse is dead) & 48 & $23.5 \%$ & $16.06 \pm 9.447$ & $57.77 \pm 19.750$ \\
\hline & 3(not married) & 15 & $7.4 \%$ & $24.53 \pm 5.527$ & $54.40 \pm 16.190$ \\
\hline \multirow[t]{5}{*}{ Education } & 1(have never attended school) & 31 & $15.2 \%$ & $19.06 \pm 9.764$ & $59.97 \pm 18.691$ \\
\hline & 2 (primary education) & 66 & $32.4 \%$ & $11.47 \pm 8.692$ & $54.62 \pm 17.601$ \\
\hline & 3(junior high school education) & 43 & $21.1 \%$ & $10.35 \pm 9.786$ & $46.93 \pm 13.165$ \\
\hline & 4(senior high school education) & 62 & $30.4 \%$ & $5.37 \pm 7.205$ & $40.02 \pm 9.915$ \\
\hline & 5(bachelor and above) & 2 & $1.0 \%$ & $0.00 \pm 0.000$ & $40.00 \pm 1.414$ \\
\hline \multirow[t]{4}{*}{ Monthly income } & $1(1000$ yuan and below) & 89 & $43.6 \%$ & $14.43 \pm 9.886$ & $55.79 \pm 44.05$ \\
\hline & $2(1000 \sim 3000$ yuan $)$ & 78 & $38.2 \%$ & $8.99 \pm 9.357$ & $44.05 \pm 13.013$ \\
\hline & $3(3000 \sim 5000$ yuan $)$ & 36 & $17.6 \%$ & $3.92 \pm 4.087$ & $44.47 \pm 15.219$ \\
\hline & $5(10000$ yuan and above $)$ & 1 & $0.5 \%$ & $0.00 \pm 0.000$ & $41.00 \pm 0.000$ \\
\hline \multirow[t]{4}{*}{ Source of income } & 1 (oneself) & 132 & $64.7 \%$ & $8.83 \pm 9.307$ & $44.51 \pm 13.301$ \\
\hline & 2 (spouse) & 26 & $12.7 \%$ & $7.31 \pm 6.632$ & $49.08 \pm 14.419$ \\
\hline & 3(children) & 40 & $19.6 \%$ & $16.13 \pm 9.952$ & $62.30 \pm 18.287$ \\
\hline & 4(society) & 6 & $2.9 \%$ & $20.83 \pm 7.757$ & $66.67 \pm 20.156$ \\
\hline \multirow[t]{2}{*}{$\begin{array}{l}\text { Type of medical } \\
\text { insurance }\end{array}$} & $\begin{array}{l}\text { 1(medical insurance for urban } \\
\text { employees) }\end{array}$ & 66 & $32.4 \%$ & $4.82 \pm 5.162$ & $45.83 \pm 14.730$ \\
\hline & $\begin{array}{l}2 \text { (medical insurance for urban and } \\
\text { rural residents) }\end{array}$ & 138 & $67.6 \%$ & $13.10 \pm 10.235$ & $50.86 \pm 17.049$ \\
\hline \multirow[t]{3}{*}{ Daily living ability } & 1(normal) & 142 & $69.6 \%$ & $8.14 \pm 9.142$ & $43.12 \pm 12.246$ \\
\hline & 2(mild living obstacle) & 37 & $18.1 \%$ & $8.73 \pm 6.119$ & $53.00 \pm 11.477$ \\
\hline & 3(obvious living obstacle) & 25 & $12.3 \%$ & $19.42 \pm 8.025$ & $68.85 \pm 16.114$ \\
\hline \multirow{4}{*}{$\begin{array}{l}\text { Number of chronic } \\
\text { diseases }\end{array}$} & $0(0$ kind $)$ & 69 & $33.8 \%$ & $8.00 \pm 9.191$ & $37.90 \pm 10.062$ \\
\hline & 1 (1 kind) & 73 & $35.8 \%$ & $8.19 \pm 8.896$ & $48.08 \pm 11.791$ \\
\hline & 2 (2 kinds) & 33 & $16.2 \%$ & $11.33 \pm 8.612$ & $54.94 \pm 13.306$ \\
\hline & 3(3 kinds and above) & 29 & $14.2 \%$ & $20.79 \pm 7.098$ & $72.59 \pm 15.394$ \\
\hline \multirow[t]{4}{*}{ Couple attachment } & 1(normal) & 93 & $45.6 \%$ & $5.86 \pm 6.924$ & $43.87 \pm 12.168$ \\
\hline & 2(mild) & 29 & $14.2 \%$ & $8.90 \pm 7.494$ & $50.76 \pm 16.745$ \\
\hline & 3(moderate-severe) & 13 & $6.4 \%$ & $13.62 \pm 10.548$ & $49.69 \pm 16.825$ \\
\hline & 4(no spouse) & 69 & $33.8 \%$ & $16.61 \pm 10.182$ & $55.72 \pm 18.992$ \\
\hline
\end{tabular}




\begin{tabular}{|c|c|c|c|c|c|}
\hline \multirow{3}{*}{$\begin{array}{c}\text { Parent- } \\
\text { child contact }\end{array}$} & (living together) & 74 & $36.3 \%$ & $11.11 \pm 9.518$ & $47.58 \pm 16.120$ \\
\hline & 2 (contact every few days) & 115 & $56.4 \%$ & $8.14 \pm 8.627$ & $49.62 \pm 16.699$ \\
\hline & 3(have no child) & 15 & $7.4 \%$ & $24.53 \pm 5.527$ & $54.40 \pm 16.190$ \\
\hline \multirow[t]{3}{*}{ Social support } & 1 (lower) & 50 & $24.5 \%$ & $20.82 \pm 8.166$ & $59.26 \pm 18.074$ \\
\hline & 2 (normal) & 109 & $53.4 \%$ & $8.30 \pm 7.727$ & $47.37 \pm 15.261$ \\
\hline & 3 (higher) & 45 & $22.1 \%$ & $4.00 \pm 6.175$ & $42.60 \pm 12.157$ \\
\hline \multirow{3}{*}{$\begin{array}{l}\text { Frequency of } \\
\text { Internet use }\end{array}$} & 0 (never) & 166 & $81.4 \%$ & $12.08 \pm 9.856$ & $51.59 \pm 16.818$ \\
\hline & 1 (occasionally) & 9 & $4.4 \%$ & $5.89 \pm 6.716$ & $40.22 \pm 8.452$ \\
\hline & 2 (often) & 29 & $14.2 \%$ & $2.34 \pm 2.832$ & $38.52 \pm 10.045$ \\
\hline \multirow[t]{3}{*}{ Depression } & 1(normal) & 123 & $60.3 \%$ & & \\
\hline & 2 (mild) & 46 & $22.5 \%$ & & \\
\hline & 3(moderate-severe) & 35 & $17.2 \%$ & & \\
\hline \multirow[t]{4}{*}{ Anxiety } & 1 (normal) & 115 & $56.4 \%$ & & \\
\hline & 2 (mild) & 40 & $19.6 \%$ & & \\
\hline & 3 (moderate) & 18 & $8.8 \%$ & & \\
\hline & 4 (severe) & 31 & $15.2 \%$ & & \\
\hline
\end{tabular}

enhance the level of anxiety and depression. In terms of type of medical insurance, the depression degree of the elderly covered by medical insurance for urban employees is much lower, the depression score of the elderly covered by medical insurance for urban and rural residents is significantly higher (while the anxiety level is just a little bit higher) than those covered by medical insurance for urban employees.

\section{Influence of Physical Health on Depression and Anxiety Status of the Elderly}

Daily living ability and the number of chronic diseases have significant influences on the depression and anxiety degree of the elderly. People with higher daily living ability and less number of chronic diseases, their depression and anxiety degrees of the elderly is lower. Among them, the depression score of the elderly with obvious living obstacles, which seriously affect the psychological and health level, is more than twice compared to those with normal status and mild living obstacles. The anxiety score of the elderly with chronic diseases of 1-2 types are basically similar, while the scores of depression with 3 or more types of chronic diseases are much higher than those with 2 types of chronic diseases. Therefore, the physical health condition of the elderly people has a very important impact on mental health. Anxiety degree increases with the number of chronic diseases.

\section{Influence of Living Environment on Depression and Anxiety Status of the Elderly}

The relationship between husband and wife, parent-children contact, as well as social support have significant influence on the depression degree of the elderly; while the relationship between husband and wife and social support have significant influence on the anxiety degree of the elderly. The weaker the couple attachment is, the higher the depression and anxiety levels tend to be. $45.6 \%$ of the surveyed elderly have normal couple relationship, with the lowest scores for anxiety and depression degree, and $33.8 \%$ of the population with no spouse have the highest scores for depression and anxiety degree, indicating the deepest feelings of loneliness. When children contact their parents every few days, the elderly has the lowest depression level, even lower than those who are living with their children, indicating that the elderly also need certain personal space, and living with their children is not necessarily the most appropriate. It suggests that children should come home every several days to visit their parents, which will reduce parents' depression level. In addition, the higher the social support level is, the lower the degrees of depression and anxiety of the elderly become.

\section{The Influence of Internet Use on Depression and Anxiety Status of the Elderly}

Due to the small number of the elderly who use the Internet in the sample, which only accounts for $18.6 \%$, the relationship between the time and purpose of Internet use and the mental health of the elderly cannot be concluded effectively. Therefore, we will not draw a conclusion on this point.

\section{Uni-variable Analysis for Top Significant Factors}

\section{The Uni-variable Analysis}

Taking depression and anxiety scores of the elderly as the dependent variables, the factors influencing mental health (age, gender, marriage, education, monthly income, source of income, type of medical insurance, daily life ability, the number of chronic disease, couple attachment, parent-child contact, social support and Internet use frequency) as the independent variables, we carry out the uni- 
variable analysis. Because the independent variables include ordered categorical and disordered categorical ones, while the dependent variable is the ordered categorical variables, uni-variance analysis requires the dependent variable to be a continuous variable obeying normal distribution. Therefore, we use chi-square test to determine whether there will be significant changes in dependent variable when independent variables take different values, namely, whether there is a significant correlation between independent variables and dependent variable. The higher the chi-square value, the greater the correlation is.

\section{The Top Three Highest Correlation Factors to Depression}

Uni-variable analysis is carried out on the depression analysis. The correlation between depression degree and various factors is shown in (Table 2). Age, marriage, education, monthly income, source of income, type of medical insurance, daily living ability, the number of chronic diseases, couple attachment, parent-child connection, social support and Internet use frequency are the significant factors influencing the depression status of elder people. However, the top three factors that have the most significant influence on depression is social support, marriage, and couple attachment. Even though number of chronic diseases and daily living ability list at the fourth and fifth in the order of significance, the degree of depression is mainly connected to how to deal with relationship between oneself and the others. This indicates that dealing well with the relationship between oneself and society or intimate relationship with spouse is a key factor for depression prevention.

Table 2. The factors which are influencing the mental health of the elderly.

\begin{tabular}{|l|c|c|c|c|}
\hline \multirow{2}{*}{ Influence factors } & \multicolumn{2}{|c|}{ Depression } & \multicolumn{2}{c|}{ Anxiety } \\
\cline { 2 - 5 } & $\chi^{2}$ & $P$ & $\chi^{2}$ & $P$ \\
\hline Age & 36.651 & 0.000 & 96.734 & 0.000 \\
\hline Gender & 2.934 & 0.231 & 6.321 & 0.097 \\
\hline Marriage & 58.047 & 0.000 & 29.891 & 0.000 \\
\hline Education & 43.829 & 0.000 & 55.848 & 0.000 \\
\hline Monthly income & 40.714 & 0.000 & 29.961 & 0.000 \\
\hline Source of income & 41.812 & 0.000 & 58.070 & 0.000 \\
\hline Type of medical insurance & 42.169 & 0.000 & 7.437 & 0.059 \\
\hline Daily living ability & 44.114 & 0.000 & 87.451 & 0.000 \\
\hline The number of chronic disease & 46.542 & 0.000 & 122.624 & 0.000 \\
\hline Couple attachment & 48.341 & 0.000 & 35.927 & 0.000 \\
\hline Parent-child contact & 33.782 & 0.000 & 5.058 & 0.536 \\
\hline Social support & 85.435 & 0.000 & 37.795 & 0.000 \\
\hline Internet use frequency & 25.932 & 0.000 & 25.750 & 0.000 \\
\hline
\end{tabular}

\section{The Top Three Highest Correlation Factors to Anxiety}

Uni-variable analysis is carried out on the anxiety analysis. As can be seen in the Table 2, age, marriage, education, monthly income, source of economy, daily life ability, number of chronic diseases, couple attachment, social support and frequency of Internet use are important factors influencing anxiety status of the elderly. Gender, Parent-child contact and type of medical insurance are not significant in the result of anxiety measurement, and are excluded from the significance group. The top three most significant factors are the number of chronic diseases, age and the daily living ability. It indicates that the top significant factors are much about the ability of daily life, and the relation between oneself, and anxiety level is more related to the concerning of a person himself and his daily life. The source of income and education level which are the fourth and fifth significant factors are also important but not as basic as the top three factors.

\section{Discussion}

\section{Special Attentions Need To Be Paid To Vulnerable Population}

Based on the analysis of demographic factors, the elderly population with advanced ages, low education level and widowed have relatively poor mental health status, which can be regarded as the vulnerable groups in the elderly population. Due to the physiological decline, the cognitive, autonomous and interpersonal skills of elderly people with advanced ages are declining, and a variety of negative events are increasing, which are prone to psychological maladjustment. Elderly people who live alone are less likely to receive timely medical care, and probably lack family life and feel much lonely. In terms of education level, the elderly with lower education level have relatively low vision and ability to adjust their anxiety state. For the vulnerable groups, they should be given more attention and special care in policy, including more concern and assistance from social and family levels, so as to promote their positive self-health management and enhance their feeling of happiness. It is necessary to further improve the system of social assistance to carry oout functions of family mutual assistance by the mode of social assistance for the aged. We vigorously advocate the traditional virtues of respecting, supporting and helping the elderly from the Chinese tradition, and develop young volunteers to participate in community support and services for them, so as to provide convenience for the elderly living alone and to reduce their loneliness. The government and academic groups should actively provide the elderly with opportunities for continuing education, help them to access the Internet and other new technologies, and encourage the elderly with low education level to fully improve their cognitive functions in a large amount of free time in order to increase their degree of happiness in life.

\section{Economic Condition of the Elderly Needs To Be Improved}

The economic basis has a significant impact on depression degree of the elderly. With higher monthly income, more stable economic source, and with the medical insurance for urban employees, the elderly have higher mental health level. With the new type of social insurance for rural residents and urban residents gradually merged, the endowment insurance coverage is quite extensive in China.

However, the growth rate of the pension for employee received by most of the elderly at the grass-roots level is far behind the currency inflation, plus the rural pension is even much lower. Adhering to the principle of 'ensuring basic care, providing broad coverage, being flexible and sustainable, the new rural social pension insurance has 
a low starting point and slow growth rate, making it difficult to meet the living needs of the elderly. In order to improve the elderly's ability to support themselves and reduce their living and mental burden, it is necessary to raise the level of endowment insurance by taking into account the currency inflation rate, the growth rate of real wages and the allowable scope of financial capacity. When talking about the economic security of the elderly, not only the basic expenditure of food and daily necessities, but also the expenditure of cultural activities that can keep the mental health of the elderly should be gradually taken into consideration. In particular, retirement pensions and basic subsistence allowances for rural residents should be increased to gradually narrow the gap between urban and rural social security levels. Besides, we can also consider encouraging capable elderly to participate in more social activities and get remuneration through their capability to improve economic income and enrich spiritual life.

\section{Primary Medical and Nursing Services Need To Be Improved}

Our results show that there is a significant correlation between the number of chronic diseases and mental health, and the daily living ability has a significant impact on the anxiety level of the elder people. The main reason for the poor mental health of the elderly is the unsatisfactory physical health condition. Accordingly, first of all, we need improve the level of medical services for the elderly at the primary level, and increase the total supply of medical services, thus to narrow the gap in the distribution of medical resources among institutions between different levels and different regions, and to provide more adequate, high-quality and equal medical supplies for the elder populations. Secondly, in terms of chronic disease management, we should take advantage of information technology and accelerate the construction of hierarchical diagnosis system; we need make full use of the doctors from community, the countryside hospital as well as family doctors to strengthen daily monitoring of the physical condition for the elderly; we need encourage the elder people to exercise and make a healthy diet; intervention and control of chronic diseases should be put into action, so as to reduce depression, anxiety degrees. Finally, for the elderly who do not have the ability to live a normal daily life, such as those who have lost the ability of selfcare, including super-aging and disabled people, we need to explore an appropriate caring mode of family and social combination.

\section{Living Environment Should Be Optimized and Social Supports Should Be Strengthened}

Family and social emotional support are indispensable for everyone, and it has significant influence for the mental health of the elder population, too. Harmonious marital relationship and frequent parent-child contact can significantly reduce the degree of depression and anxiety of the elderly. More participation in social activities can also protect the elderly from depression and anxiety.

Since family interactions and social activities are very important to the elderly, we need to take a multi-pronged approach to strengthen the emotional support for the elderly from both family and society. First of all, the public attitude should be changed in recognizing the problems related to mental disorders, more open and active discussion of related topics in public shall be promoted, and mental health knowledge should be popularized, such that the elderly should be encouraged to discover mental health problems as early as possible, and take the initiative to seek medical treatment. Secondly, we should promote activities to enrich the cultural life of the elderly in the community and township levels, in order to eliminate their loneliness and depression, to help them to develop a sense of belonging and enjoy the benefits brought by society progress. Thirdly, efforts should be made to develop social work organizations of community staffs with mental health knowledge to communicate with the elderly regularly, and especially for the elderly in vulnerable groups. In such a way, the elderly with mental problems or potential anxiety and depression tendencies could be provided with better social environment support.

\section{Conclusion}

Through uni-variable analysis of depression and anxiety in the elderly, this study found that depression degree was affected by 12 factors among which the top three significant factors are social support, marriage and couple attachment, which are mainly related to the relationship between oneself and others. The anxiety level was affected by 10 factors, among which the top three significant factors are number of chronic diseases, age and daily living ability, which are mainly related to how to deal with one in the basic daily life. Improvement of their mental health requires the participation of individuals, families, society and the government.

\section{References}

1. Chen Lixin, Chen Gong, Zheng Xiaoying (2008) Depression symptoms and influencing factors of widowed elderly in Beijing. Chinese Journal of Gerontology, 28: 696-698.

2. Chen Minxia (2018) Research on the influence of income on self-rated health status of the elderly. Master Thesis. Capital University of Economics and Business.

3. Han Xueqing, Feng Feng, Chen Jian, Shang Lan, Li Juan (2005) Definition of dementia value assisted by daily living ability scale. Chinese tissue engineering study 9: 13-15.

4. Li Juan (2018) Effects of family interaction and social activity participation on depression in the elderly. Master Thesis. Capital University of Economics and Business.

5. Zhai Xiaoyan, Li Chunhua, Wei Hong, Wang Dahua (2010) Preparation of attachment questionnaire for elderly couples. Psychological Development and Dducation 26: 197-204.

6. Derogatis LR (1992) SCL-90-R, administration, scoring and procedures manual-II for the Revised version and other instruments of the Psychopathology Rating Scale Series. Clinical Psychometric Research by Townson.

7. Zung, WWK (1965) A self-rating depression scale. Archives of General Psychiatry 12: $63-70$.

8. Zung WW (1969) A cross-cultural survey of symptoms in depression. Am J Psychiatry 126: 116-121. [crossref]

9. Lawton MP, Brody EM (1969) Assessment of older people: self-maintaining and instrumental activities of daily living. Gerontologist 9: 179-186. [crossref]

10. Wu Zhenyun (2013). Connotation, evaluation and research overview of mental health of the aged. Chinese Journal of Gerontology 33: 799-801.

11. Wu Wenyuan (2001) Anxiety self-rating scale. See Zhang Zuoji (Ed.). Manual of behavioral medicine scale (pp.124-125). Beijing: China medical electronic audio and video publishing company.

12. Yang Jingjing, Zheng Yong (2010) Intergenerational relationship: a new perspective on mental health of the elderly. Chinese Journal of Gerontology 30: 2875-2878.

13. Liu Jiwen, li Fuye, Lian Yulong (2008) Reliability and validity of social support rating scale. Journal of Xinjiang Medical University 31: 1-3.

14. Liu Mengqi (2018) Research on influencing factors of mental health of the elderly in China and policy Suggestions -based on the follow-up survey of health and oldage care in China in 2015. Master Thesis. Zhejiang University? 
Yin Zhang, Chuang Shi, Yinyun Li (2019) Depression and Anxiety Analysis from Elder Population in A Beijing Rural Area

15. Qi Yuling, Gao Hang, Zhang Xiumin (2017). Mental health status of elderly people in urban communities and its influencing factors. Nursing research 31: 26-28.
16. Xiao Shuiyuan (1994) Theoretical basis and research application of «Social Support Rating Scale». Clinical Psychiatry medical Journal 4: 98-99.

\section{Citation:}

Yin Zhang, Chuang Shi, Yinyun Li (2019) Depression and Anxiety Analysis from Elder Population in A Beijing Rural Area. Ageing Sci Ment Health Stud Volume 3(3): $1-7$. 